Applied Mathematical Sciences, Vol. 8, 2014, no. 165, 8251 - 8255

HIKARI Ltd, www.m-hikari.com

http://dx.doi.org/10.12988/ams.2014.411912

\title{
Detection and Estimation of Jumps in Discrete Time
}

\author{
Denis Bosq \\ Sorbonne Universités, UPMC Univ Paris 06, F-75005 \\ EA 3124, Laboratoire de Statistique Théorique et Appliquée \\ 4 place Jussieu, Paris, France \\ Delphine Blanke \\ Avignon University \\ Laboratoire de Mathématiques d'Avignon, EA2151 \\ 33 rue Louis Pasteur, F-84000 Avignon, France \\ Dedicated to Professor Marius Stoka on the occasion of his $80^{\text {th }}$ birthday \\ Copyright (c) 2014 Denis Bosq and Delphine Blanke. This is an open access article \\ distributed under the Creative Commons Attribution License, which permits unrestricted \\ use, distribution, and reproduction in any medium, provided the original work is properly
} cited.

\begin{abstract}
We consider $n$ random functions defined on $[0,1]$ and admitting a finite number of jumps. By using discrete observations we intend to detect the instants of jumps. As an Application, we obtain estimators of the intensity of jumps with an exponential rate.
\end{abstract}

Mathematics Subject Classification: 62M

Keywords: Processes with jumps, Estimation of intensity, Sampled processes

\section{Introduction}

Existence of jumps in random functions is very frequent: they appear in meteorology [cf 2], electricity consumption [cf 1, 7], finance [cf 9] and, more generally, 
in the compound Poisson process. Another interesting example is the mistral gust [see 8].

The natural space for studying these jumps is the space $D=D[0,1]$ of cadlag real functions defined over $[0,1]$. Some facts concerning $D$ will appear in Section 2.

Now, let $X$ be a $D$-valued random variable admitting instants of jumps $t_{1}, \ldots, t_{k}$ all distinct and belonging to $(0,1)$, then, one may set

$$
\Delta_{j}=\left|X\left(t_{j}\right)-X\left(t_{j}-\right)\right|
$$

with

$$
0<E \Delta_{j}<\infty, 1 \leq j \leq k .
$$

The problem is to detect $\left(t_{j}, j=1, \ldots, k\right)$ given i.i.d. copies of $X_{i}$ observed at the instants $\frac{l}{q_{n}}, 0 \leq l \leq q_{n}, 1 \leq i \leq n$, where $l$ and $q_{n} \geq 1$ are integers.

An Hölder regularity condition allows to detect $t_{1}, \ldots, t_{k}$ at an exponential rate (cf Section 2).

Then, by using the detectors, one may again estimate $E \Delta_{1}, \ldots, E \Delta_{k}$ at an exponential rate; the main tool being the Bernstein's inequality.

Note that it is possible to replace the fixed instants by independent random instants. One may also extend the results to $D$-valued ARMA processes.

Concerning the proofs and the simulations, they will appear in Blanke and Bosq [5] ; see also Bosq [6] and Blanke and Bosq [4] for further results on this topic.

\section{Detecting fixed jumps}

Let $D$ be the space of cadlag real functions defined over [0,1]. Since the uniform norm \|. $\|$ implies non separability, it is preferable to choose the Skorohod metric $d$ [see 3 , for details]. Thus, $D$ will be equipped with the $\sigma$-algebra $\mathcal{D}$ associated with $d$.

Now, let $X$ be a random variable which is $D$ valued and $\mathcal{D}$ measurable. We suppose that $X$ has $k$ distinct instants of jumps $t_{1}, \ldots, t_{k}$ belonging to $(0,1)$ and with random intensity

$$
\Delta_{j}=\left|X\left(t_{j}\right)-X\left(t_{j}-\right)\right|, 1 \leq j \leq k .
$$

We want to estimate $E \Delta_{j}$ from the i.i.d. copies $\left(X_{i}\left(\frac{l}{q_{n}}\right), 0 \leq l \leq q_{n}, 1 \leq i \leq n\right)$ where $l$ and $q_{n} \geq 1$ are integers and $q_{n} \rightarrow \infty$ as $n \rightarrow \infty$.

Then, since the data are discrete, we need a regularity assumption. Below the $t_{j}$ 's are classified in increasing order: $t_{1}^{*}<\cdots<t_{k}^{*}$ : 
Assumption 2.1. For each $1 \leq i \leq n$ :

$$
\left|X_{i}(t)-X_{i}(s)\right| \leq M_{i}|t-s|^{\alpha}
$$

where $(s, t) \in\left[0, t_{1}^{*}\left[{ }^{2} \cup \cdots \cup\left[t_{k}^{*}, 1\right]^{2}, 0<\alpha \leq 1, E M_{i}^{p}<\infty, p \geq 1\right.\right.$.

Note that the fractional Brownian motion and the Ornstein-Uhlenbeck process with jumps satisfy Assumption 2.1 (with $n=1$ ).

In order to detect the instants of jumps we consider the following approximation of $t_{j}$ which is valid for $q_{n}$ large enough:

$$
0<\frac{l_{j n}-1}{q_{n}}<t_{j} \leq \frac{l_{j n}}{q_{n}}:=t_{j n}, 1 \leq j \leq k
$$

also we set

$$
\bar{\Delta}_{l n}=\frac{1}{n} \sum_{i=1}^{n}\left|X_{i}\left(\frac{l}{q_{n}}\right)-X_{i}\left(\frac{l-1}{q_{n}}\right)\right|, 1 \leq l \leq q_{n} .
$$

Finally we may and do suppose that

$$
0<E \Delta_{k}<\cdots<E \Delta_{1}<\infty
$$

and we consider the detectors

$$
\hat{t}_{1 n}=\frac{1}{q_{n}} \arg \max _{1 \leq l \leq q_{n}} \bar{\Delta}_{l n}:=\frac{\hat{l}_{1 n}}{q_{n}}
$$

if $k \geq 2$ we put

$$
\hat{t}_{2 n}=\frac{1}{q_{n}} \arg \max _{1 \leq l \leq q_{n}, l \neq \hat{l}_{1 n}} \bar{\Delta}_{l n}:=\frac{\hat{l}_{2 n}}{q_{n}}
$$

and so on...

The following assumption implies that $\hat{t}_{1 n}, \ldots, \hat{t}_{k n}$ are almost surely unique:

Assumption 2.2. The distribution of $\bar{\Delta}_{l n}$ is continuous, $1 \leq l \leq q_{n}$.

Now, in order to obtain a convergence rate we suppose that we have:

Assumption 2.3. For some $c>0, E(\exp (c\|X\|)<\infty$.

Then, we get the following statement concerning the detector:

Proposition 2.4. If Assumption 2.1, 2.2 and 2.3 hold, then

$$
P\left(\bigcup_{j=1}^{k}\left\{\hat{t}_{j n} \neq t_{j n}\right\}\right)=\mathcal{O}\left(\exp -n c_{k}\right), c_{k}>0 .
$$


Corollary 2.5. Under the same assumptions, we obtain:

$$
P\left(\bigcup_{j=1}^{k}\left|\hat{t}_{j n}-t_{j}\right|>\frac{1}{q_{n}}\right)=\mathcal{O}\left(\exp -n c_{k}\right), c_{k}>0 .
$$

Consequently, almost surely for $n$ large enough, the distance between $\hat{t}_{j n}$ and $t_{j}$ is not greater than $\frac{1}{q_{n}}$. Note that this result cannot be improved since the rate of discretization is $\frac{1}{q_{n}}$.

\section{Estimating the intensity of jumps}

In order to estimate the intensity of jumps we use $\hat{t}_{j n}$ and set

$$
\hat{D}_{j n}=\frac{1}{n} \sum_{i=1}^{n}\left|X_{i}\left(\hat{t}_{j n}\right)-X_{i}\left(\hat{t}_{j n}-\frac{1}{q_{n}}\right)\right|, \quad 1 \leq j \leq k .
$$

First we get

Proposition 3.1. If Assumption 2.2 and 2.3 hold, we have

$$
P\left(\hat{D}_{j n}-E\left|X\left(\hat{t}_{j n}\right)-X\left(\hat{t}_{j n}-\frac{1}{q_{n}}\right)\right| \geq \eta\right)=\mathcal{O}(\exp -n c(\eta)),
$$

$1 \leq j \leq k, \quad(c(\eta)>0)$

And finally

Corollary 3.2. Under Assumption 2.1, 2.2 and 2.3, one obtains

$$
P\left(\left|\hat{D}_{j n}-E \Delta_{j}\right| \geq \eta\right)=\mathcal{O}(\exp -n d(\eta))
$$

$1 \leq j \leq k, \quad(d(\eta)>0)$

Then, it follows that almost surely for $n$ large enough, $\left|\hat{D}_{j n}-E \Delta_{j}\right|=$ $\mathcal{O}\left(\sqrt{\frac{\log n}{n}}\right), 1 \leq j \leq k$. Some simulations show that the approximation of the intensities of jumps are satisfactory [cf 5].

\section{References}

[1] A. Antoniadis, X. Brossat, J. Cugliari, and J.-M. Poggi. Prévision d'un processus à valeurs fonctionnelles en présence de non stationnarités. Application à la consommation d'électricité. J. SFdS, 153(2):52-78, 2012. 
[2] P. Besse, H. Cardot, and D. Stephenson. Autoregressive forecasting of some climatic variation. Scandinavian J. Statist., 27(4):673-687, 2000.

[3] P. Billingsley. Convergence of probability measures. Wiley Series in Probability and Statistics: Probability and Statistics. John Wiley \& Sons Inc., New York, second edition, 1999.

[4] D. Blanke and D. Bosq. Exponential bounds for intensity of jumps. Math. Methods Statist, to appear, 2014. 22 pages.

[5] D. Blanke and D. Bosq. Observing jumps in discrete time. Applications. In preparation, 2014.

[6] D. Bosq. Estimating and detecting jumps. Applications to D[0,1]-valued linear processes. Springer, 2014. Festschrift in honour of Paul Deheuvels. To appear.

[7] L. El Hajj. Théorèmes limites pour les processus autorégressifs à valeurs dans D[0,1]. PhD thesis, Université Paris 6, Paris, Décembre 2013.

[8] V. Jacq, P. Albert, and R. Delorme. Le mistral. Quelques aspects des connaissances actuelles. La météorologie, 50:30-38, août 2005.

[9] P. Tankov and E. Voltchkova. Jump-diffusion models: a practitioner's guide. Banque et Marchés, 99:1-24, 2009.

Received: September 1, 2014; Published: November 25, 2014 